\title{
The Characteristics Approach to the Measurement of Population Aging
}

Warren C. Sanderson (wsanderson@notes.cc.sunysb.edu)

Sergei Scherbov (scherbov@iiasa.ac.at)

\section{Approved by}

Wolfgang Lutz

Leader, World Population Program

June 24,2013

Interim Reports on work of the International Institute for Applied Systems Analysis receive only limited review. Views or opinions expressed herein do not necessarily represent those of the Institute, its National Member Organizations, or other organizations supporting the work. 


\section{Contents}

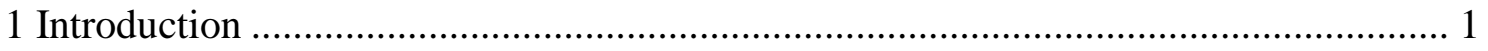

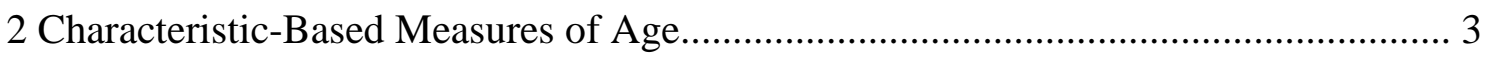

3 Characteristics-Based Measures of Population Aging .............................................. 6

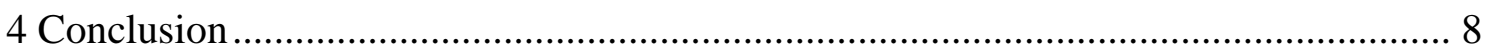

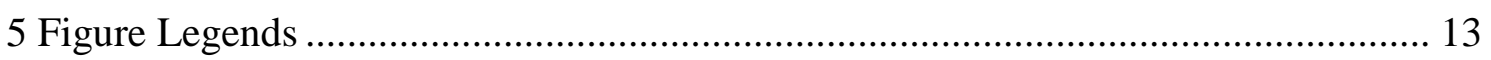

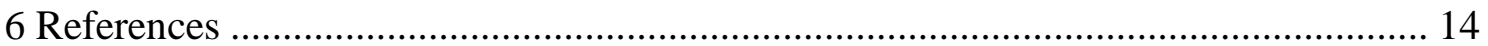




\section{Abstract}

Traditional measures for aging have been in use for many decades. They are widely applied by interested parties from scientists to politicians and are used in many national and international organizations. These measures provide the fundamental inputs into the analysis of population aging and into other related fields. Because they do not take into account changes in people's characteristics such as improvements in life expectancy and health, these measures are becoming increasingly inappropriate for both scientific and policy analysis. There is now an emerging new paradigm that considers multiple characteristics of people including, but not limited to, their chronological age. In this paper, we provide the history of the characteristics approach to the measurement of population aging and examine its basic mathematical structure. Using examples from Japan, Lithuania, Norway, and the United States we show that this paradigm provides us with new and insightful measures of population aging. 


\section{Acknowledgments}

This research was supported by a grant from the European Research Council (ERC2012-AdG 323947-Re-Ageing). 


\section{About the Authors}

Sergei Scherbov is Project Leader at the International Institute for Applied Systems Analysis, and Research Group Leader at the Vienna Institute of Demography, Austrian Academy of Sciences.

Warren C. Sanderson is Professor of Economics and Professor of History at Stony Brook University in New York. He is also an Associate Research Scholar at IIASA's World Population Program. 


\title{
The Characteristics Approach to the Measurement of Population Aging
}

\author{
Warren C. Sanderson and Sergei Scherbov
}

\section{Introduction}

Conventional studies of population aging focus only on chronological age and produce limited and, in many respects, biased results. The UN's reference volume, World Population Ageing, 1950-2050 (United Nations 2001), contains measures of population aging, all of which depend on only one characteristic of people, their chronological age. This implicitly assumes that other characteristics relevant for the study of aging do not change over time and space. But clearly, they do. For example, 65 year olds today generally have higher remaining life expectancies and are healthier than their earlier counterparts. 65 year olds, who were eligible for full public pensions in the many OECD countries a few decades ago, will not be eligible for them a few decades in the future. The study of population aging is useful because important characteristics of people change with age, but age-specific characteristics also change over time and differ from place to place. Focusing on one aspect of the changes of the characteristics of populations, but not on all the others provides a limited picture of aging, one that is often not appropriate for either scientific study or policy analysis.

There is an emerging more general paradigm in the field of population aging, one that considers multiple characteristics of people not just their chronological age. Remaining life expectancy, health, disabilities rates, cognitive functioning, and eligibility for a full public pension are also important for understanding population aging.

The new paradigm first emerged in Ryder (1975). Ryder wrote:

"To the extent that our concern with age is what it signifies about the degree of deterioration and dependence, it would seem sensible to consider the measurement of age not in terms of years elapsed since birth but rather in terms of the number of years remaining until death. ... We propose that some arbitrary length of time, such as 10 years, be selected and that we determine at what age the expectation of life is 10 years, that age to be considered the point of entry into old age ...” (p. 16)

Ryder used his characteristic-based definition of the threshold of old age to tabulate those ages for the "West" family of Coale-Demeny model life tables (Coale and Demeny 1966). In addition, he computed the proportions old, using his definition, for a variety of stable populations in the "West" group.

Although Ryder's reasoning was revolutionary, its importance was realized only slowly. Ryder himself made no further use of it and the next paper that applied it came 
almost a decade later. There could be a number of reasons for its initially limited development. First, his discussion appeared in an article about stable populations, not in an article about aging. In 1975, when his article appeared, there was little interest in the demographic community in population aging. The major policy concern was rapid population growth in less developed countries. Years later, when concern about population aging began to increase, Ryder's research on stable populations was not an obvious reference. Second, Ryder's interest seemed limited to defining a more meaningful threshold of old age. For many demographers this was not a pressing issue. The convention that people became elderly at age 65 seemed both simple and sensible. In essence, Ryder was providing an answer to a question that almost no one was asking.

Siegel and Davidson (1984) were the first to apply Ryder's insight to observations. They used two remaining life expectancies, 10 and 15 years, to define ages at which old age began for the United States in Census years from 1920 to 1980. Like Ryder, they used those years to compute proportions of the population who were "old" according to those definitions. Interestingly, the proportion old was the same in 1980 as it was in 1940. Siegel and Davidson also realized that remaining life expectancy as a characteristic could be used for more than defining the old age threshold. They suggested that it could also be used in government programs, such as in indexing the age at the receipt of a full Social Security pension in the United States.

In a pattern that characterized the slow emergence of the new paradigm, this insight of Siegel and Davidson was forgotten. When the same idea arose around a quarter of a century later it was independently reinvented in Shoven (2007), which made reference neither to Ryder (1975) nor to Siegel and Davidson (1984).

The intellectual ancestor of Shoven (2007) and the related articles Shoven (2008) and Shoven and Goda (2010) was not the demographer Ryder, but the economist Fuchs. Fuchs (1984) was interested in the proportion of the population who were elderly. He tabulated data for the US using three different definitions: (1) the proportion of the population $65+$ years old, (2) the proportion of the population $65+$ years old, who would die in the succeeding five years, and (3) the proportion of the population 65+ years old who are not in the labor force. Definitions (2) and (3) supplement chronological age with characteristics of people that change over time. Although Fuchs wrote nine years after Ryder, his analysis was not as complete. Ryder computed a threshold age at which people became elderly and used this to produce a new measure of population aging. Fuchs suggested new measures of the proportion elderly without thinking about how this related to the concept of age.

The Siegel and Davidson (1984) estimates of proportions elderly in the United States have been updated (Siegel 1993; Siegel 2011), but this research never cited the alternative definitions suggested by Fuchs. With the exception of Siegel's publications, Ryder's ideas about age and aging remained unused until Heigl (2002) proposed an interesting application. Heigl wanted to obtain a quantitative measure of the changes over time in the active life expectancy of the elderly. To do this, he used Ryder's threshold age for becoming elderly and computed active life expectancy from that age forward. Heigl was the first to propose a measure that combined two time-varying agespecific characteristics. According to the Web of Science (Thompson Reuters), Heigl's article, which was written in German, has, up to now, never been cited. 
When we wrote Sanderson and Scherbov (2005), we were unaware of the previous literature. In that article, we realized that age could be computed both backwards using the number of previous birthdays and forwards using remaining life expectancy. We used our forward-looking age to compute what we subsequently called prospective median ages, median ages adjusted for changes in life expectancies. We also presented a new version of the conventional old age dependency ratio, where the threshold ages at the beginning and the end of the working age period were adjusted for changes in life expectancies. Determining prospective median ages is different in an important way from Ryder (1975) and Siegel and Davidson (1984). In both of those, the level of a characteristic was chosen and this determined a series of ages associated with that level. In determining the prospective median age, there is no fixed level of a characteristic that can be used.

Subsequently, we have written a number of articles (Lutz et al. 2008; Sanderson and Scherbov 2008; Sanderson and Scherbov 2007; Sanderson and Scherbov 2010) that have expanded this line of inquiry and others have been engaged in this approach as well (for example, Skirbekk et al. 2012). However, the methodology still lacks a name, a core set of equations and definitions, and a consciousness that the literature taken together constitutes of new paradigm in the field of measuring population aging. We call the new paradigm "the characteristics approach to the measurement of population aging”. It has taken decades for it to fully emerge, but now that policies with respect to aging populations are growing in interest and importance, its applications can be especially useful.

The remainder of the paper is organized as follows. In Section 2, we discuss characteristic-based measures of age. In Section 3, we present new characteristic-based measures of population aging. The final section contains our concluding thoughts.

\section{Characteristic-Based Measures of Age}

Aging is a multidimensional phenomenon, but it is frequently viewed using measurement techniques that take only a single dimension into account, chronological age. Chronological age has been used as a simple summary measure of people's health, productivity, cognitive ability, disability status, pension eligibility and many other aspects of their lives. But the relationship that each of these characteristics has to age is changing. For example, people 65 years old age now are healthier than they were in the past and have lower rates of severe disabilities (McLaughlin et al. 2012; Christensen et al. 2009). In 28 out of the 34 OECD countries pension age reforms are now in progress or are planned to start soon (OECD 2012). A view of aging based only on chronological age alone is incomplete and inadequate.

In our characteristics-based approach to study population aging, we use $a$ to denote an age in the index year, $t$. Let the characteristic schedule $C(a, t)$ be a function of chronological age and time. For simplicity we omit place and subgroup subscripts here. For example, a characteristic schedule could be derived from a table of remaining life expectancies. If $C(a, t)$ is continuous and monotonic in $a$, we can invert it to obtain the age associated with a particular value, $k$, of the characteristic at time $t$. We define constant-characteristic ages, $\propto$-ages, as those where the level of the characteristic is constant.

$\propto_{k, t}=A(k, t)$ 
where $\propto_{k, t}$ is the age where the level of the characteristic is $k$ at time $t$ and $A(. .$.$) is the$ inverse of the characteristic schedule. For example, if our characteristic was a remaining life expectancy of 15 years for Americans of both sexes in 2010, the corresponding $\alpha$ age would be 71 and 1 month, the age at which that remaining life expectancy was attained.

The characteristics approach to the measurement of population aging includes all the conventional measures, but is far more general. For concreteness, in this paper we focus on four characteristics, chronological age, remaining life expectancy, the mortality rate, and the proportion of adult person-years lived after a particular age. The same approach allows the use of many other characteristics as well. Each of these characteristics has a particular interpretation for the study of population aging. We include chronological age here for two reasons: (1) to show how natural it is to embed conventional measures in the generalized framework, and (2) to provide a quantitative benchmark against which to assess the importance of including other characteristics. We include remaining life expectancy here because it can be used to judge when people become elderly, as Ryder and others have done. We include the mortality rate here because it can be used as a rough, but easily measurable ordinal indicator of the health of a group of older people. Finally, we include the proportion of adult person-years lived after a given age because it can be used to construct a simple hypothetical demographically indexed public pension system.

Ryder (1975) applied what we now call $\propto$-ages to show how the chronological age at which people became elderly changes in stationary populations with different life expectancies. Siegel and Davidson (1984) computed $\alpha$-age trajectories for the US showing how the chronological age at the onset of becoming elderly changed over time. In cases such as these, we call the resulting trajectories "transition trajectories" because they provide ages at which a transition is made from one state to another. Transition trajectories can be used to answer a number of questions. How old do you have to be to be considered “old”? One approach to answering this question is to follow Ryder (1975) and stipulate that people are old when they get close to end of their lives, or in other words, when their remaining life expectancy, ex, falls below a threshold value. With an appropriate choice of the threshold, the corresponding $\alpha$-age can be used to determine the onset of "old age".

Another approach is to use a health indicator to mark the entrance to old age. Health is such a complex phenomenon, but a rough and readily accessible measure would be to associate population health at given ages in an ordinal way with the level of the age-specific mortality rate. In this case, $\alpha$-ages based on the life table measure $m_{x}$ would provide ages of comparable population health across space and time (Cutler et al. 2007; Vaupel 2010; Fuchs 1984) and could also be used to mark the transition to old age.

Another important transition is the one in which people become eligible for a full public pension. $\alpha$-ages can also help us here because they allow us to easily specify a simple public pension system where the fraction of adult person-years spent eligible for a pension remains constant. This public pension system is equitable in the sense that the ratio of years of pension to years in the working ages remains fixed, even as life expectancy changes. We call the ratio of person-years lived at age $\mathrm{x}$ and beyond to the number of person-years lived from age $20, \frac{T_{x}}{T_{20}}$, the "life course ratio" because it allows 
fruitful links to life course studies (Lee and Goldstein 2003). In the special case, where the life course ratio is the constant fraction of adult person-years in which people are eligible for a pension, the corresponding $\alpha$-age provides an easily understood measure that defines the age at pension eligibility and can therefore be used to inform discussions of pension age changes.

Ryder chose the remaining life expectancy at which he thought old age began according to his intuition. In characteristic approach to the study of population aging, we study a number of different characteristics of people and if we were to specify threshold characteristic levels intuitively for each of them, we could encounter inconsistencies. In Figure 1, we show the $\alpha$-age transition trajectories for the onset of old age using remaining life expectancy and using health status. We also show how a demographically indexed pension age changes. When specifying threshold levels of various characteristics to be used in studying population aging, it is best to determine them consistently and comparatively. We implement this in Figure 1 by setting the values of the characteristics at the levels observed for 65 year olds in their countries in 1965.

Figure 1 has panels for four countries, Japan, Lithuania, Norway and the US. In each we show the three $\alpha$-age transition trajectories and a horizontal line for age 65 . By construction all four line coincide at age 65 in 1965. Two features are evident. First, Lithuania has had a pattern of aging distinctly different from those of the other three countries. Instead of having rising ages at the transition to old age after 1970, it has fluctuating $\alpha$-ages with no clear trend. With little improvements in survivorship at older ages, the onset of old age did not change much. Lithuania's experience is similar to that of many other Eastern European countries. Second, since 1970 in Western European countries, the health-based $\alpha$-age rises faster than the other two. This indicates that if, after 1970, pension ages had been adjusted according to our demographically indexed pension system, the health of people when they first retired would be improving. The age at eligibility for a full public pension rises more slowly than the other two $\alpha$-ages. For example, in our demographically indexed public pension plan, the age at eligibility for a full public pension in the US would have risen at a rate of about one and a third months per year over the period 1965-2010.

$\alpha$-ages have many more uses in addition to producing interesting transition trajectories. They have been computed for the ratio of life expectancy to life expectancy at birth, $\mathrm{e}_{\mathrm{x}} / \mathrm{e}_{0}$, and ratio of life expectancy at birth to life expectancy at $20, \mathrm{e}_{\mathrm{x}} / \mathrm{e}_{20}$ (Shoven and Goda 2010; Siegel 2011). Shoven and Goda (2010) have used these as well as remaining life expectancy and single-year mortality rates to study the effects of how age is incorporated into various pieces of US legislation. $\alpha$-ages have also been computed for self-reported health and specific impairments (Cutler et al. 2007).

Thus far, we have treated the case where $\alpha$-ages are based on constant characteristics. $\alpha$-ages can also be used to study situations where the level of the characteristic is changing over time. To do this, we can rewrite equation (1) as:

$\alpha_{k_{t}, T}=A\left(k_{t}, T\right)$,

where $k_{t}$ is the level of the characteristic in year $t$ and $T$ is some fixed year used as a reference. In this case, the $\alpha$-age is the chronological age in the reference years that has the same level of the characteristic observed in year $t$. Such $\alpha$-ages have been used to 
study median ages, accounting for changes in life expectancies (Sanderson and Scherbov 2005; Lutz et al. 2008; Sanderson and Scherbov 2008).

The age-specific characteristics of people differ across countries as well as change over time. Cross-country comparisons can be made by specifying the characteristic level for a particular country and time, and varying characteristic schedules by country (equation 1) or by specifying the characteristic schedule for a particular country and time and varying the characteristic by country (equation 2) (Sanderson and Scherbov 2008).

\section{Characteristics-Based Measures of Population Aging}

In the conventional framework, age itself is not an object of study. If people have always grown old at age 65 and if they will always grow old at age 65 in the future, there is nothing to study. The age at the onset of old age is fixed forever. However, if our interest is in the capabilities, functioning, and health of people, then equivalent ages become of substantive interest. Investigations into $\alpha$-ages are based on changing characteristic schedules and are independent of the age structure of the population.

While studies of age deal only with changing characteristic schedules, our studies of population aging combine the changing characteristic schedules and age structures of populations. The conventional approach to measuring population aging ignores those changing characteristics. For example, one of the most common measures of the population aging is the proportion of the population 65+ years old. It takes an invariant chronological age at which people enter old age (65 years old in this case) and interacts it with the age structures of populations. Our expanded approach to the study of population aging incorporates $\alpha$-ages instead of fixed chronological ages.

Conventional measures of population aging have the form

$M_{t}^{c}=f(S(a, t), H(a))$,

where $M_{t}^{c}$ is a conventional measure of aging at time $t, S(a, t)$ is the age structure of the population at time $t$, and $H$ is a matrix of age-specific characteristics. $S(a, t)$ can be a vector of the number of people by age or a matrix of the number of people by age, sex, and other informative dimensions. The key feature of conventional measures is that $H$ is independent of time. Age structures of populations are allowed to change over time, but the characteristics of people are not.

The new measures that we analyze here have the form

$M_{t}^{n}=g(S(a, t), H(a, t))$,

where $M_{t}^{n}$ is a new characteristics based measure at time $t$ and where the matrix $H(a, t)$ includes time-varying age-specific characteristics.

Two sorts of measures are common. We introduce the terms elder proportions and elder ratios to denote the entire families of measures. Elder proportions have the form $\frac{\sum_{a=0}^{\omega} s_{a, t} h_{a, t}}{\sum_{a=0}^{\omega} s_{a, t}}$, where $s_{a, t}$ is the population at age $a$ at time $t, \omega$, is the highest possible age, and $h_{a, t}$ are age-specific characteristics. When $h_{a, t}$ is an indicator variable that takes on the value of unity when age is $65+$, we obtain the standard proportion of the population $65+$ years old. 
Figure 2 presents elder proportions for the countries, where the $h_{a, t}$ are indicator variables based on the $\alpha$-ages shown in Figure 1. Although the proportions $65+$ rise in all countries, the most recent observations of the characteristic-based elder proportions in Norway and the US are lower than they were in 1970. Adjusting for the changing characteristics of the population allows us to see that in some ways the populations of Norway and the US had been growing functionally younger.

Characteristics that have been used in the literature include an indicator variable that takes on the value of unity when the age is above the $\alpha$-age associated with $e_{x}$ (Sanderson and Scherbov 2005; Lutz et al. 2008; Sanderson and Scherbov 2008), and the proportion of people expected to die within 5 years, among those 65+ (Fuchs 1984).

Elder ratios differ from elder proportions because people with the characteristic associated with the elderly are excluded from the denominator. A special subset of elder ratios, $\alpha$-old age dependency ratios, is based on indicator variables constructed from threshold $\alpha$-ages.

$\alpha-O A D R(t)=\frac{\sum_{a=\bar{a}}^{\omega} s_{a, t} h_{a, t}}{\sum_{a=\bar{a}}^{\omega} s_{a, t}\left(1-h_{a, t}\right)}$,

where $\bar{a}$ is some initial age, often 15 or 20 . When $h_{a, t}$ is an indicator variable that takes on the value of unity when age is $65^{+}$, we obtain the standard old age dependency ratio (OADR).

In Figure 3, we present the conventional OADR and $\propto$-OADRs for the four countries based on the three threshold $\alpha$-ages shown in Figure 1. The conventional OADR in Japan increased rapidly, but all the other $\alpha$-OADRs show much more modest aging. In the US, the conventional OADR rises from 1965 onward, while the adjusted ones generally fall. The characteristics based approach to aging provides a natural framework for seeing these differences.

Elder ratios that use levels of the characteristics themselves and not indicator variables include the Adult Disability Dependency Ratio (ADDR) (Sanderson and Scherbov 2010), which uses time-varying rates of severe disability, and the CognitionAdjusted Dependency Rate (CADR) (Skirbekk et al. 2012), which uses a measure of cognition for people $50+$.

Elder relationships have the form $\frac{\sum_{a=\bar{a}}^{\omega} s_{a, t} h_{a, t}}{\sum_{a=\bar{a}}^{\omega} s_{a, t} j_{a, t}}$, where $h_{a, t}$ and $j_{a, t}$ refer to two different characteristics. We provide an example of an elder relationship in Figure 4. There $h_{a, t}$ and $j_{a, t}$ are indicator variables based on the $\propto$-ages in Figure 1 . The $h_{a, t}$ are set to unity when age is greater than the $\propto$-age threshold associated with a remaining life expectancy of 15 years and the $j_{a, t}$ are set to unity when age is greater than the $\propto$-age threshold associated with pension receipt (in the context of our idealized pension system). Figure 4 shows that these ratios have been decreasing over time after the mid1960s, even for Lithuania. This indicates that over time fewer and fewer people receiving pensions (under our idealized pension system) would be considered old. Elder relationships are natural quantities to compute in the framework of a characteristicbased approach to the study of population aging, but, to our knowledge, none have previously appeared in the literature.

Elder proportions, elder ratios, and elder relationships are only three ways of incorporating changing characteristics into the study of population aging. There are 
clearly others, as the measure in Heigl (2002) illustrates. The hallmark of the characteristics approach to the measurement of population aging is the consistent use of changing characteristic schedules together with changing age structures, regardless of the exact way in which the two are combined.

\section{Conclusion}

The conventional approach to the study of population aging considers only a single characteristic of people, their chronological age. This simplification is appropriate under some circumstances, but it is certainly not necessary. People have multiple characteristics and these characteristics have been changing and are likely to continue changing in ways that are central to the study of population aging. The new paradigm allows age itself to be conceptualized more generally, through the use of constantcharacteristic ages. The characteristics based approach also allows the construction of new measures of aging, including measures that show how various aspects of aging change relative to one another. This is especially important for the analysis of policies with respect to pension ages.

The approach to the study of population aging based only on chronological age has been around for over a century. It was extremely useful for most of its lifetime, but the new paradigm presented here is more appropriate for dealing with the kinds of demographic changes that we expect to experience in the twenty-first century. 

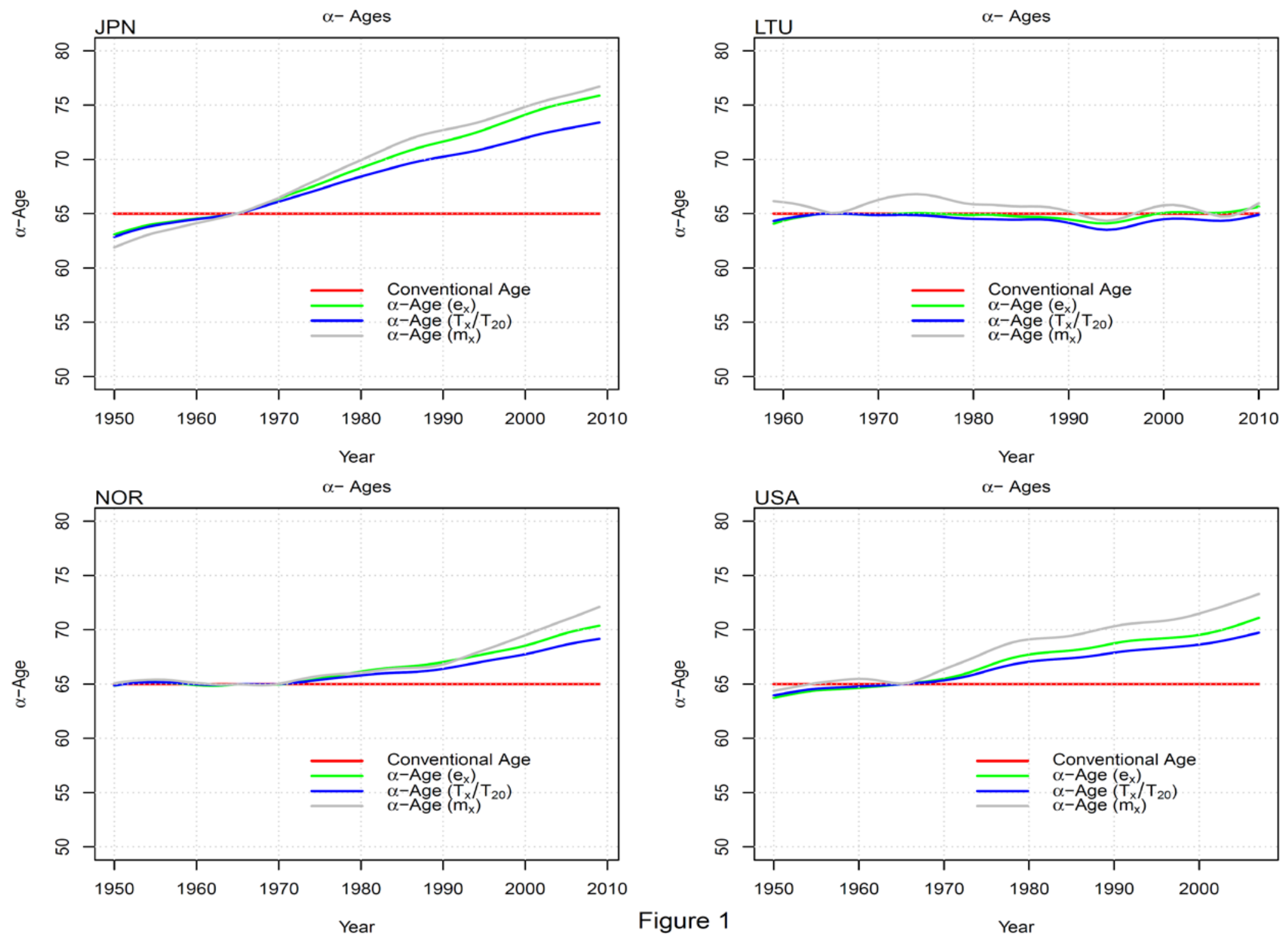

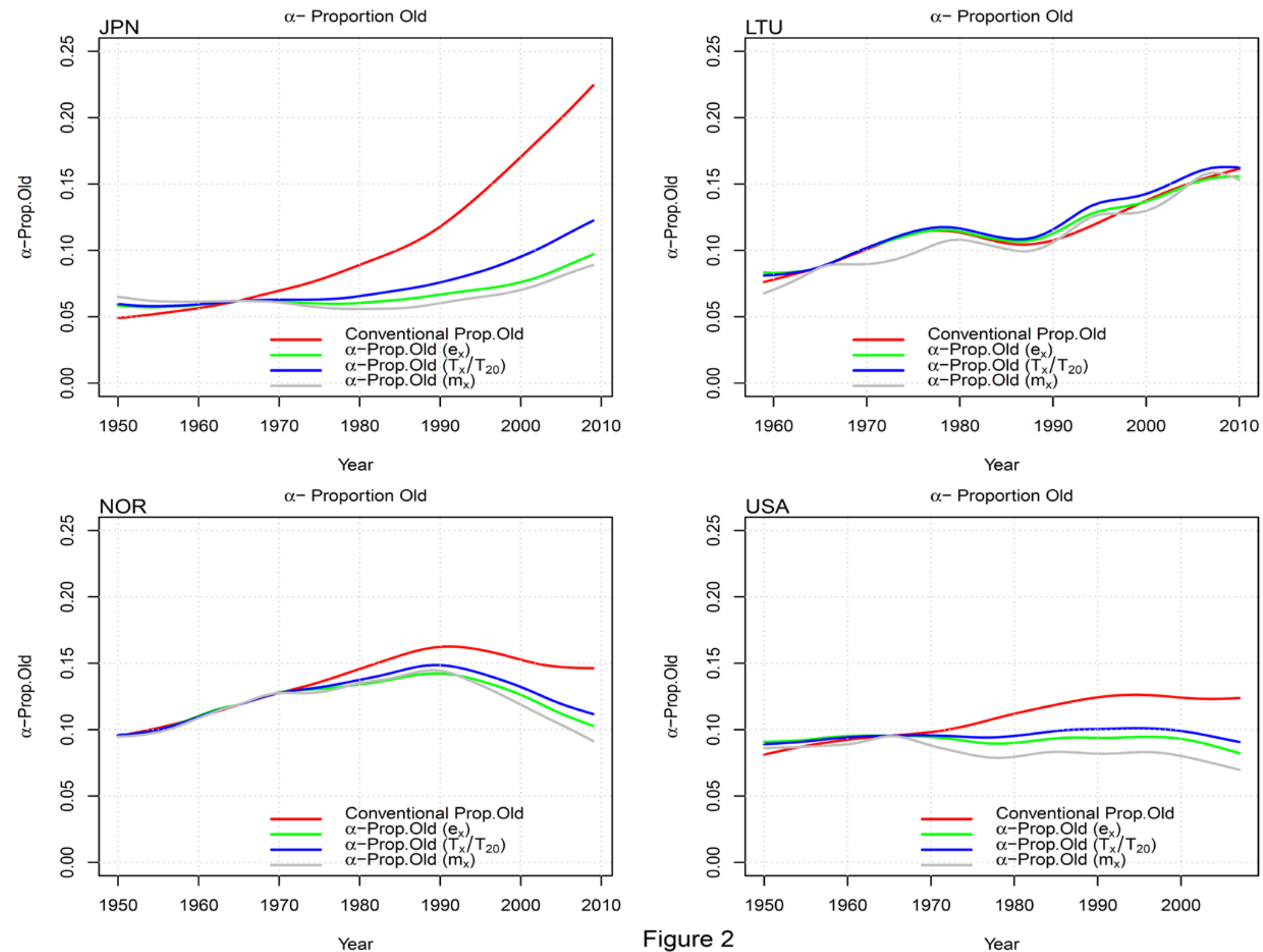

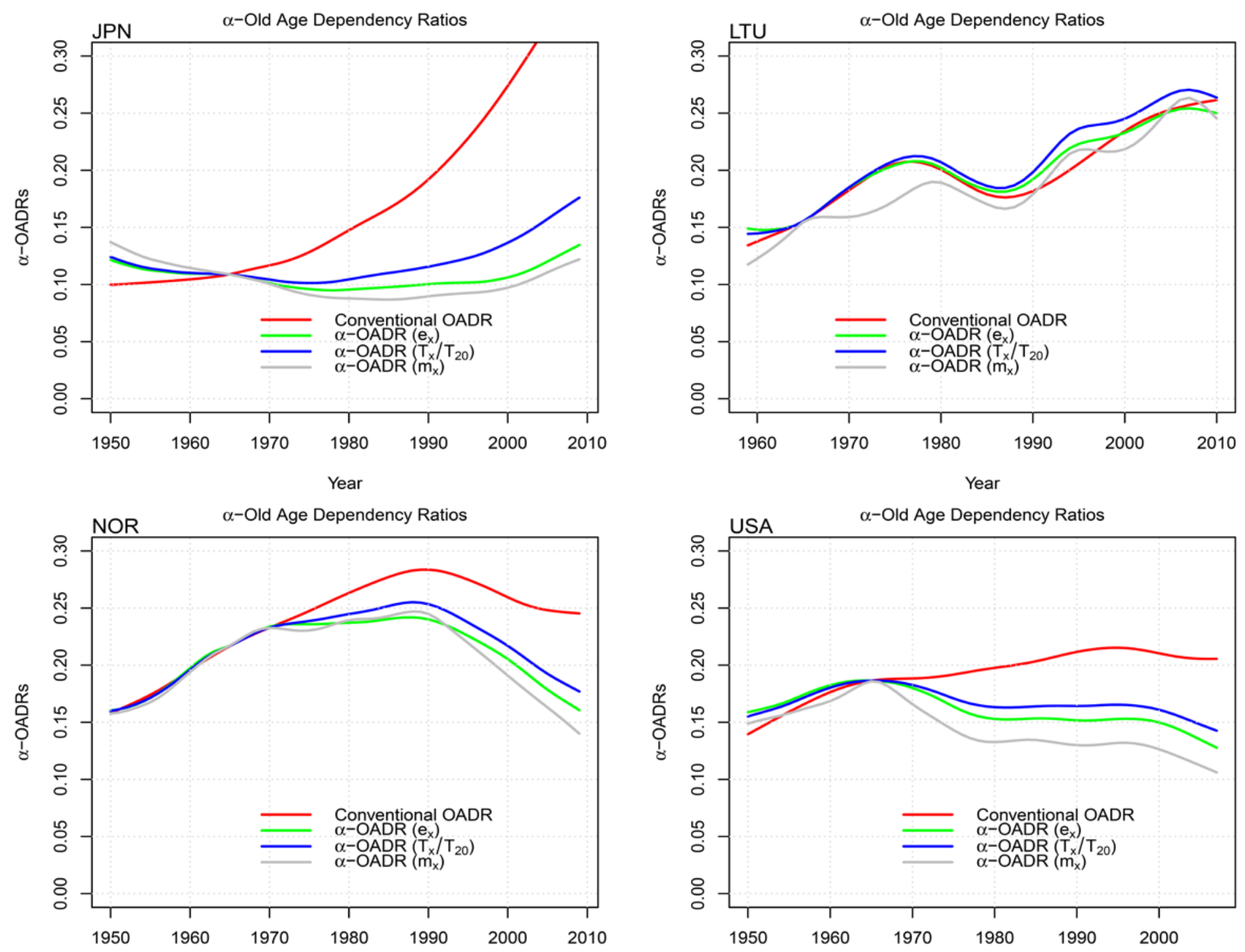

Figure 3

Year 


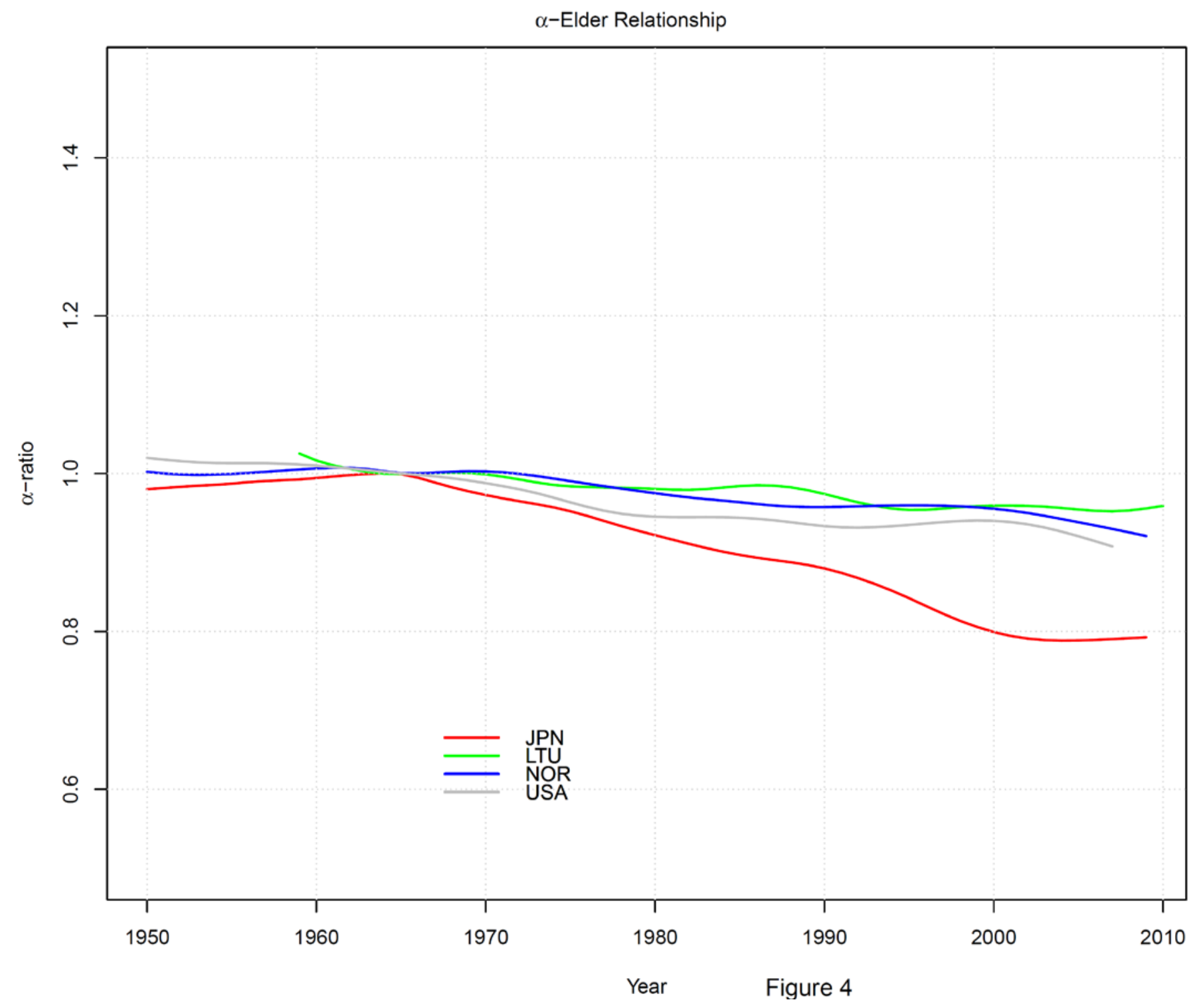




\section{Figure Legends}

Figure 1: Three characteristic based $\propto$-ages

$\propto$-ages associated with 3 characteristics, remaining life expectancy, $e_{x}$, the mortality rate, $m_{x}$, and the life course ratio, $\frac{T_{x}}{T_{20}}$, for Japan, Lithuania, Norway, and the US, most recent data from 1950 onwards.

Source: Human Mortality Database (accessed February 1, 2012) and authors' calculations.

Note: Smoothing was done with splines, keeping the observation for 1965 equal to age 65.

Figure 2: Elder proportions for Japan, Lithuania, Norway and the US

Elder proportions computed using age 65 and the $3 \propto$-ages in Figure 1for Japan, Lithuania, Norway and the US, most recent data from 1950 onwards.

Source: Human Mortality Database (accessed February 1, 2012) and authors' calculations.

Note: Smoothing was done with splines.

Figure 3: $\propto$-Old Age Dependency Ratios for Japan, Lithuania, Norway and the US $\propto$-Old Age Dependency Ratios computed using age 65 and the $3 \propto$-ages in Figure 1 for Japan, Lithuania, Norway and the US, most recent data from 1950 onwards.

See Source and Note for Figure 2.

Figure 4: Elder Relationships for Japan, Lithuania, Norway and the US

Elder Relationships computed using proportion of the population at or above the $\propto$-age associated with ex in the numerator and the proportion of the population at or above the $\propto$-age associated with life course ratio in the denominator, for Japan, Lithuania, Norway and the US, most recent data from 1950 onwards.

See Source and Note for Figure 2. 


\section{References}

Christensen, K., G. Doblhammer, R. Rau and J. W. Vaupel. 2009. Ageing populations: the challenges ahead. Lancet 374(9696): 1196-1208.

Coale, A. J. and P. Demeny. 1966. Regional Model Life Tables and Stable Populations. New York: Academic Press.

Cutler, D., J. Liebman, M. Shepard and S. Smyth. 2007. An Expaned Model of Health and Retirement. Retirement Research Center Paper. Cambridge, MA.: National Bureau of Economic Research.

Fuchs, V. R. 1984. “Though much is taken”: Reflections on aging, health, and medical care. Milbank Memorial Fund Quarterly-Health and Society 62(2): 143-166.

Heigl, A. 2002. Active life expectancy: Concepts and a new model approach. Zeitschrift für Gerontologie und Geriatrie 35(6), 519-527.

University of California, Berkley, CA, \& Max Planck Institute for Demographic Research (Germany). (n.d.). Human Mortality Database. Retrieved February 1, 2012, from http://www.mortality.org.

Lee, R. and J. R. Goldstein. 2003. Rescaling the Life Cycle: Longevity and Proportionaltiy. In: R. Carey and S. Tuljapurkar (eds.) Life Span: Evolutionary, Ecologicial and Demographic Perspectives. New York: The Population Council.

Lutz, W., W. C. Sanderson and S. Scherbov. 2008. The coming acceleration of global population ageing. Nature 451(7179): 716-719.

McLaughlin, S. J., A. M. Jette and C. M. Connell. 2012. An examination of healthy aging across a conceptual continuum: Prevalence estimates, demographic patterns, and validity. The Journals of Gerontology. Series A, Biological Sciences and Medical Sciences 67(7): 783-789.

OECD. 2012. OECD Pensions Outlook 2012. Paris: OECD.

Ryder, N. B. 1975. Notes on Stationary Populations. Population Index 41(1): 3-28.

Sanderson, W. C. and S. Scherbov. 2008. Rethinking age and aging. Population Bulletin 63(4): 3-16.

Sanderson, W. C. and S. Scherbov. 2005. Average remaining lifetimes can increase as human populations age. Nature 435(7043): 811-813.

Sanderson, W. C. and S. Scherbov. 2007. A new perspective on population aging. Demographic Research 16: 27-57.

Sanderson, W. C. and S. Scherbov. 2010. Remeasuring aging. Science 329(5997): 12871288.

Shoven, J. 2007. New Age Thinking: Alternative Ways of Measuring Age, Thier Relationship to Labor Force Participation, Government Policies and GDP. NBER Working Paper. Cambridge, Mass.: National Bureau of Economic Research.

Shoven, J. 2008. Adjusting Government Policies for Age Inflation. NBER Working Paper. Cambridge, Mass.: National Bureau of Economic Research.

Shoven, J. and G. Goda. 2010. Adjusting Government Policies for Age Inflation. In: J. Shoven (ed.) Demography and Economics. Chicago: University of Chicago Press.

Siegel, J. S. 1993. A Generation of Change: A Profile of America's Older Population. New York: Russell Sage Foundation.

Siegel, J. S. 2011. The Demography and Epidemiology of Human Health and Aging. New York: Springer. 
Siegel, J. S. and M. Davidson. 1984. Demographic and Socioeconomic Aspects of Aging in the United States. Current Population Reports P-23, 138. Washington, D.C.: U.S. Government Printing Office.

Skirbekk, V., E. Loichinger and D. Weber. 2012. Variation in cognitive functioning as a refined approach to comparing aging across countries. Proceedings of the National Academy of Sciences of the United States of America 109(3) 770-774.

Thompson Reuters. Web of Science. Retrieved February 1, 2012, from http://thomsonreuters.com/web-of-science/

United Nations 2001. World Population Ageing: 1950-2000. New York: United Nations.

Vaupel, J. W. 2010. Biodemography of human ageing. Nature 464(7288): 536-542. 